\title{
Polysomnography versus limited respiratory monitoring and nurse-led titration to optimise non- invasive ventilation set-up: a pilot randomised clinical trial
}

\author{
Maxime Patout, ${ }_{1,2,3}$ Gill Arbane, ${ }^{1,3}$ Antoine Cuvelier, ${ }^{2}$ Jean Francois Muir, ${ }^{2}$ \\ Nicholas Hart, ${ }^{1,3,4}$ Patrick Brian Murphy ${ }^{1,3,4}$
}

- Additional material is published online only. To view please visit the journal online (http://dx.doi.org/10.1136/ thoraxjnl-2017-211067).

${ }^{1}$ Lane Fox Respiratory Unit, Guy's and St Thomas' NHS Foundation Trust, London, UK ${ }^{2}$ Normandie Univ, UNIRouen, EA3830-GRHV, Institute for Research and Innovation in Biomedicine (IRIB), Rouen University Hospital, Service de Pneumologie, Oncologie thoracique et Soins Intensifs Respiratoires, Rouen, France ${ }^{3}$ Lane Fox Clinical Respiratory Physiology Research Centre, Guy's and St Thomas' NHS Foundation Trust, London, UK ${ }^{4}$ Centre for Human, Aerospace and Physiological Sciences, King's College London, London, UK

\section{Correspondence to}

Dr Maxime Patout, Lane Fox Respiratory Unit, St-Thomas Hospital, London SE1 7EH, UK; maxime.patout@chu-rouen.fr

Received 21 September 2017 Revised 13 March 2018 Accepted 19 March 2018 Published Online First 30 March 2018
Check for updates

To cite: Patout M, Arbane $\mathrm{G}$, Cuvelier A, et al. Thorax 2019;74:83-86.

\section{ABSTRACT}

Polysomnography (PSG) is recommended for noninvasive ventilation (NIV) set-up in patients with chronic respiratory failure. In this pilot randomised clinical trial, we compared the physiological effectiveness of NIV set-up guided by PSG to limited respiratory monitoring (LRM) and nurse-led titration in patients with COPDobstructive sleep apnoea (OSA) overlap. The principal outcome of interest was change in daytime arterial partial pressure of carbon dioxide $\left(\mathrm{PaCO}_{2}\right)$ at 3 months Fourteen patients with daytime $\mathrm{PaCO}_{2}>6 \mathrm{kPa}$ and body mass index $>30 \mathrm{~kg} / \mathrm{m}^{2}$ were recruited. At 3 months, $\mathrm{PaCO}_{2}$ was reduced by $-0.88 \mathrm{kPa}(95 \% \mathrm{Cl}-1.52$ to $-0.24 \mathrm{kPa})$ in the LRM group and by $-0.36 \mathrm{kPa}(95 \% \mathrm{Cl}$ -0.96 to $0.24 \mathrm{kPa}$ ) in the PSG group. These pilot data provide support to undertake a clinical trial investigating the clinical effectiveness of attended limited respiratory monitoring and PSG to establish NIV in patients with COPD-OSA overlap.

Trial number Results, NCT02444806.

\section{INTRODUCTION}

Patients with COPD-obstructive sleep apnoea (COPD-OSA) overlap syndrome have a greater incidence of chronic respiratory failure compared with patients with OSA alone. ${ }^{1}$ Non-invasive ventilation (NIV) has been demonstrated as an effective treatment ${ }^{2}$ and current guidelines recommend polysomnography (PSG) accompanied by waveform analysis and retrospective NIV modification on the following day to direct NIV titration. ${ }^{3}$ However, access to PSG is limited and, combined with a rising numbers of patients requiring investigation for sleep-disordered breathing, clinical capacity is unable to match demand. Simple overnight transcutaneous measurements of gas exchange with nurse-led titration according to a protocol has been used as an alternative to PSG-directed set-up. ${ }^{4-6}$ The aim of the current study was to determine the physiological efficacy of limited respiratory monitoring (LRM) using transcutaneous oxi-capnometry and nurse-led titration, compared with PSG and retrospective NIV modification, for the set-up of NIV in patients with chronic respiratory failure secondary to COPD-OSA overlap.

\section{METHODS}

The study protocol was approved by Guy's and St Thomas' Research Ethics Committee (14/EM/1257) and the trial registered in a publically accessible database (NCT02444806).

\section{Inclusion and exclusion criteria}

Patients naive to home NIV use were recruited if they met the following criteria: diagnosis of COPD as defined by the Global Initiative for Chronic Obstructive Lung Disease criteria, ${ }^{7}$ OSA defined by a $4 \%$ oxygen desaturation index $>7.5$ events/hour or an apnoea-hypopnoea index $>5$ events/hour, a daytime arterial $\mathrm{PaO}_{2}$ of carbon dioxide $\left(\mathrm{PaCO}_{2}\right)>6 \mathrm{kPa}$ and a body mass index (BMI) $>30 \mathrm{~kg} / \mathrm{m}^{2}$. Exclusion criteria included decompensated respiratory failure with a $\mathrm{pH}<7.35$, inability to tolerate NIV ( $<4$ hours usage during titration), contraindication to NIV, pregnancy, age $<18$ years and any significant physical or psychiatric comorbidity that would prevent adherence to the trial protocol. Patients were randomised 1:1 to have NIV set-up using LRM or PSG. All overnight titrations in the PSG arm were performed by a single specialist trained respiratory physician. In the LRM arm, the overnight NIV titration was performed by a specialist trained nurse from the ward clinical team, which was not always the same member of staff. Final assessment was performed 3 months after NIV set-up.

\section{Outcomes}

Principal outcome of interest was daytime arterial partial pressure of carbon dioxide $\left(\mathrm{PaCO}_{2}\right)$ at 3 months. Other relevant outcomes were daytime $\mathrm{PaO}_{2}$, adherence to ventilation (measured hours of use recorded by device software), health-related quality of life (measured by severe respiratory questionnaire; SRI), sleep quality (measured by Pittsburgh Sleep Quality Index; PSQI) and physiological efficacy of NIV demonstrated by change in neural respiratory drive.

Full methodology including the nurse-led titration protocol and NIV modification protocol and statistical analysis plan have been placed in the online (supplementary material and methods, pages 1-4 OLS; figure E1, page 9 OLS).

\section{RESULTS}

\section{Baseline}

Recruitment was performed between 25 February and 7 November 2015. Forty-two patients were screened for study participation and 14 included 
Table 1 Baseline characteristics of study population

\begin{tabular}{|c|c|c|c|}
\hline & $\begin{array}{l}\text { Overall population }(n=14) \\
\text { Mean } \pm \text { SD/median }(\mathrm{IQR}) / \text { number } \\
(\%)\end{array}$ & $\begin{array}{l}\text { LRM } \\
(n=7) \\
\text { Mean } \pm S D / \text { median }(\text { IQR }) / \text { number } \\
(\%)\end{array}$ & $\begin{array}{l}\text { PSG } \\
(\mathrm{n}=7) \\
\text { Mean } \pm S D / \text { median }(\mathrm{IQR}) / \text { number } \\
(\%)\end{array}$ \\
\hline Age (years) & $62.4 \pm 9.3$ & $64.1 \pm 11$ & $60.7 \pm 8$ \\
\hline Gender (male) & $11(79 \%)$ & $6(86 \%)$ & $5(71 \%)$ \\
\hline \multicolumn{4}{|l|}{ Anthropometrics } \\
\hline BMI $\left(\mathrm{kg} / \mathrm{m}^{2}\right)$ & $37.2 \pm 5.7$ & $35.5 \pm 4$ & $38.9 \pm 7$ \\
\hline Neck circumference $(\mathrm{cm})$ & $47.3 \pm 5$ & $45.3 \pm 5.5$ & $49.4 \pm 3.7$ \\
\hline \multicolumn{4}{|l|}{ COPD severity } \\
\hline Medical Research Council Dyspnoea scale* (/5) & $4.7 \pm 0.5$ & $4.9 \pm 0.4$ & $4.6 \pm 0.5$ \\
\hline Borg Scalet (/10) & $2.5(0.6-3.8)$ & $3.0(0.0-5.0)$ & $2.0(0.5-3.0)$ \\
\hline $\mathrm{FEV}_{1}(\mathrm{~L})$ & $1.00 \pm 0.7$ & $0.93 \pm 0.2$ & $1.07 \pm 0.9$ \\
\hline $\mathrm{FEV}_{1}(\%$ predicted $)$ & $30.1 \pm 15.3$ & $31.4 \pm 12.3$ & $28.7 \pm 18.8$ \\
\hline FVC (L) & $1.7 \pm 0.8$ & $1.6 \pm 0.3$ & $1.8 \pm 1.2$ \\
\hline FVC (\% predicted) & $40.8 \pm 15.4$ & $42.3 \pm 10.7$ & $39.3 \pm 19.8$ \\
\hline $\mathrm{FEV}_{1} / \mathrm{FVC}$ & $56.7 \pm 10$ & $58.2 \pm 12.2$ & $55.3 \pm 8.1$ \\
\hline Smoking history $(\mathrm{p} / \mathrm{y})$ & $42(31-58)$ & $40(20-58)$ & $50(35-60)$ \\
\hline No of acute exacerbations over the last 12 months & $1.5(0.0-2.0)$ & $2.0(0.0-2.0)$ & $1.0(0.0-3.0)$ \\
\hline \multicolumn{4}{|l|}{ Daytime arterial blood gas on room air } \\
\hline $\mathrm{pH}$ & $7.38 \pm 0.03$ & $7.39 \pm 0.03$ & $7.37 \pm 0.02$ \\
\hline $\mathrm{PaO}_{2}(\mathrm{kPa})$ & $7.72 \pm 0.72$ & $7.36 \pm 0.8$ & $8.08 \pm 0.4$ \\
\hline $\mathrm{PaCO}_{2}(\mathrm{kPa})$ & $7.18 \pm 0.78$ & $7.03 \pm 0.4$ & $7.33 \pm 1$ \\
\hline \multicolumn{4}{|l|}{ Health-related quality of life questionnaires } \\
\hline COPD Assessment Tool‡ (/40) & $24.4 \pm 4.7$ & $22.9 \pm 2$ & $25.9 \pm 6$ \\
\hline Severe Respiratory Insufficiency Questionnaire§ (/100) & $48.4(40.9-59.7)$ & $47.3(40.2-62.1)$ & $49.5(28.3-61.1)$ \\
\hline \multicolumn{4}{|l|}{ Sleep-related questionnaires } \\
\hline Epworth Sleepiness Scaleף & $10(7-14)$ & $7(5-16)$ & $11(8-15)$ \\
\hline Pittsburgh Sleep Quality Index** & $11( \pm 3.2)$ & $11(8-14)$ & $12(8-13)$ \\
\hline \multicolumn{4}{|l|}{ Baseline sleep study (respiratory polygraphy) } \\
\hline $4 \%$ Oxygen desaturation index (/h) & $36.4(26.8-53.3)$ & $33.6(17.1-46.7)$ & $51.1(26.0-55.0)$ \\
\hline Apnoea-hypopnoea index (/h) & $19.7(17.6-23.4)$ & $19.3(11.6-37.8)$ & $19.7(12.8-25.1)$ \\
\hline Mean $\mathrm{SpO}_{2}(\%)$ & $88.2(86.9-88.9)$ & $87.4(85.0-89.0)$ & $88.6(87.4-89.4)$ \\
\hline Percentage of time spent with $\mathrm{SpO}_{2}<90 \%$ & $66.4(41.1-81.2)$ & $73.8(35.7-89.0)$ & $63(39.7-73.0)$ \\
\hline Mean $\mathrm{tcCO}_{2}(\mathrm{kPa})$ & $7.8 \pm 0.9$ & $7.4 \pm 0.6$ & $8.1 \pm 1$ \\
\hline
\end{tabular}

* Medical Research Council Dyspnoea score- $0-5$ with higher levels indicating more severe limitations due to dyspnoea.

†Borg score $-0-10$ categorical rating of perceived exertion with higher ratings indicating more severe dyspnoea.

‡COPD assessment tool—higher scores indicate worse quality of life.

§Severe Respiratory Insufficiency Questionnaire-higher scores indicate better quality of life.

IEpworth Sleepiness Score- $0-24$ scale with higher scores indicating more severe daytime somnolence.

**Pittsburgh Sleep Quality Index (PSQI)—0-21. PSQI above 5 indicates poor quality of sleep.

$\mathrm{BMI}$, body mass index; LRM, limited respiratory monitoring; $\mathrm{PSG}$, full polysomnography; $\mathrm{SpO}_{2}$, oxygen saturation of pulsatile haemoglobin; tc $\mathrm{CO}_{2}$, transcutaneous carbon dioxide.

(online supplementary figure E2, page 10 OLS). No patients withdrew or were lost to follow-up. Three (43\%) patients in each group were established on long-term oxygen therapy. Baseline characteristics are shown in table 1. Length of stay for baseline assessments and NIV set-up was similar between both groups; 2 (2-2) nights in the LRM arm versus 2 (2-3) nights in the PSG arm. NIV settings were similar between both groups (table 2). Visual analogue scales of ease of falling asleep, comfort of sleep and comfort of breathing were similar between both groups.

\section{Outcomes of ventilation at 3 months}

At trial completion (3 months), there was a reduction in $\mathrm{PaCO}_{2}$ of $-0.88 \mathrm{kPa}(95 \% \mathrm{CI}-1.52$ to $-0.24 \mathrm{kPa})$ in the LRM group and of $-0.36 \mathrm{kPa}(95 \% \mathrm{CI}-0.96$ to $0.24 \mathrm{kPa})$ in the PSG group. Mean adherence was 4.4 hours/night (95\% CI 2.8 to 6.0 ) in the LRM group and 2.3 hours/night (95\% CI 0.91 to 3.8 ) in the PSG group. Within the 7 days preceding final evaluation, NIV was used for more than 4 hours for 4.7 nights (95\% CI 2.7 to 6.7 ) in the LRM group and 0.7 nights $(95 \%$ CI 0.0 to 2.4 ) in the PSG group. Changes in other relevant outcome variables were similar (table 2).

In the overall study population, there was a significant improvement in daytime $\mathrm{PaCO}_{2}$ (mean difference of $-0.62(95 \%$ CI -1.02 to -0.22$)$ ), in SRI (mean difference 10.8 (95\% CI -2.6 to 19.0)), Epworth Sleepiness Scale (mean difference $-1.9(95 \% \mathrm{CI}-3.6$ to -0.1$)$ ) and PSQI (mean difference -4.3 
Table 2 NIV set-up data and secondary outcomes 3 months after initiation of NIV

\begin{tabular}{|c|c|c|c|}
\hline & $\begin{array}{l}\text { Overall population } \\
(\mathrm{n}=14) \\
\text { Mean } \pm \mathrm{SD} / \text { median }(\mathrm{IQR}) / \\
\text { mean difference }(95 \% \mathrm{Cl})\end{array}$ & $\begin{array}{l}\text { LRM } \\
(\mathrm{n}=7) \\
\text { Mean } \pm \text { SD/median }(\mathrm{IQR}) / \\
\text { mean difference }(95 \% \mathrm{CI})\end{array}$ & $\begin{array}{l}\text { PSG } \\
(\mathrm{n}=7) \\
\text { Mean } \pm \text { SD/median }(\mathrm{IQR}) / \\
\text { mean difference }(95 \% \mathrm{Cl})\end{array}$ \\
\hline IPAP $\left(\mathrm{cmH}_{2} \mathrm{O}\right)$ & $25.8 \pm 2.9$ & $25.7 \pm 3.5$ & $26.0 \pm 2.3$ \\
\hline $\operatorname{EPAP}\left(\mathrm{cmH}_{2} \mathrm{O}\right)$ & $10 \pm 1.9$ & $9.1 \pm 1.9$ & $10.9 \pm 1.6$ \\
\hline Pressure support $\left(\mathrm{cmH}_{2} \mathrm{O}\right)$ & $15.9 \pm 2.9$ & $16.6 \pm 2.8$ & $15.1 \pm 3$ \\
\hline BUR (breaths/min) & $15.7 \pm 1.7$ & $15.1 \pm 1.1$ & $16.3 \pm 2.1$ \\
\hline $\mathrm{Ti}(\mathrm{s})$ & $1.06 \pm 0.1$ & $1.06 \pm 0.1$ & $1.06 \pm 0.1$ \\
\hline Average use per day (hours) & $3.5(1.5$ to 4.6$)$ & $4.5(3.5$ to 5.6$)$ & 2 (1 to 3.6$)$ \\
\hline Patient triggered breaths (\%) & 37 (31 to 50$)$ & 34 (31 to 44$)$ & 48 (15 to 56$)$ \\
\hline Expiratory Vt (mL) & $654 \pm 142$ & $653 \pm 125$ & $657 \pm 169$ \\
\hline Respiratory rate (/min) & $17.1 \pm 1.2$ & $16.8 \pm 1.1$ & $17.4 \pm 1.4$ \\
\hline Average non-intentional leak (L/min) & 18.1 (15.1 to 26.2$)$ & 19.6 (14.8 to 38.8$)$ & $17.5(10.1$ to 24.9$)$ \\
\hline $\begin{array}{l}\text { Residual apnoeic event according to NIV built-in } \\
\text { software }\end{array}$ & $2.2(1.6$ to 7.5$)$ & $2.8(1.5$ to 8.7$)$ & $2.0(0.8$ to 21.1$)$ \\
\hline Change in Severe Respiratory Insufficiency Questionnaire & $10.8(2.6$ to 19.0$)$ & $14.0(-1.0$ to 28.9$)$ & $7.6(-3.6$ to 19.0$)$ \\
\hline Change in COPD Assessment Tool & $-3.9(-7.8$ to 0.1$)$ & $-1.3(-7.1$ to 4.6$)$ & $-6.4(-12.8$ to 0.0$)$ \\
\hline Change in Epworth Sleepiness Scale & $-1.9(-3.6$ to -0.1$)$ & $-1.9(-3.2$ to -0.5$)$ & $-1.9(-5.7$ to 2.0$)$ \\
\hline Change in Pittsburgh Sleep Quality Index & $-4.3(-6.5$ to -2.1$)$ & $-6.1(-9.2$ to -3.1$)$ & $-2.4(-5.5$ to 0.7$)$ \\
\hline $\begin{array}{l}\text { Subjective sleep quality } \\
\text { (1: poor/2: average/3: good) }\end{array}$ & 2.2 (2 to 2.7$)$ & 2.5 (2 to 2.8$)$ & 2.1 (1.5 to 2.5$)$ \\
\hline Total sleep time (min) & 380 (352 to 420$)$ & 346 (223 to 433) & 400 (375 to 465 ) \\
\hline Wake after sleep onset (min) & 144 (105 to 181$)$ & 142 (42 to 210$)$ & 144 (109 to 174) \\
\hline Sleep latency (min) & 11 (7 to 24$)$ & 11 (6 to 21$)$ & 15 (6 to 37$)$ \\
\hline Sleep efficiency (\%) & 64 (56 to 77$)$ & 61 (57 to 71$)$ & 71 (55 to 81) \\
\hline
\end{tabular}

Objective sleep quality measured by 2-week actigraphy following NIV set-up.

BUR, back-up rate; EPAP, expiratory positive airway pressure; IPAP, inspiratory positive airway pressure; LRM, limited respiratory monitoring; NIV, non-invasive ventilation; PSG, full polysomnography; Ti, inspiratory time.

(95\% CI -6.5 to -2.1$)$ ).

Results detailing the NIV set-up and the nested physiological study are available in the online supplement (results pages 4-6 OLS).

\section{DISCUSSION}

This pilot randomised physiological effectiveness trial demonstrated that LRM using transcutaneous oxi-capnometry and nurse-led NIV titration, in patients with COPD-OSA with chronic respiratory failure, resulted in a greater fall in daytime $\mathrm{PaCO}_{2}$ at 3 months compared with gold-standard PSG monitoring and retrospective NIV modification. The improvements in health-related quality of life and daytime somnolence were in line with previous published data and of a similar magnitude to those achieved in patients with obesity-related respiratory failure. $^{8}$

Despite randomisation, patients allocated to the PSG-directed titration had more severe COPD-OSA overlap when compared with patients allocated to LRM-directed titration. BMI, neck circumference, smoking history and baseline daytime $\mathrm{PaCO}_{2}$ were all numerically higher in the PSG arm. These differences may have contributed to the higher EPAP in the PSG group, which in turn could be responsible for adverse pulmonary mechanics with reduced respiratory muscle unloading and subsequent poor comfort and reduced adherence. However, the comfort scores at NIV set-up were the same. The lower NIV adherence in the PSG arm may explain, in part, the lower reduction of daytime $\mathrm{PaCO}_{2}$ at follow-up. ${ }^{9}$ The small sample size of the study prevents the completion of an adjusted analysis to try and account for the differences in NIV adherence or any other baseline differences between the two groups. The results from this pilot proof-of-concept clinical trial need to be interpreted with caution and are hypothesis generating rather than hypothesis testing, which is the role of a multicentre clinical trial.

Despite these limitations, our data support the use of transcutaneous combined oxi-capnometry monitoring, in combination with a nurse-led titration protocol to safely establish NIV in patients with chronic respiratory failure secondary to COPD-OSA overlap syndrome. Both LRM and PSG groups achieved similar clinical effectiveness in terms of change in daytime $\mathrm{PaCO}_{2}$, health-related quality of life and sleep quality. These data support the safety and efficacy of limited respiratory monitoring and nurse-led titration as a simpler alternative approach to PSG for home NIV set-up. The next step will be to proceed to a multicentre clinical trial that will investigate the clinical and cost-effectiveness of limited respiratory monitoring with a nurse-led titration protocol. This is in line with the current direction of home NIV delivery with the use of auto-titrating devices, which have been shown to be safe and effective in patients with COPD-OSA overlap syndrome. ${ }^{6}$ Indeed, the built-in ventilator software may offer additional data, including $\mathrm{Vt}$, leak flow and volume, triggered breaths ${ }^{10}$ and residual events. ${ }^{11}$ 
Correction notice This article has been corrected since it was published Online First. A sentence in the Abstract and the Outcome section has been corrected.

Contributors MP: conception of the work, acquisition, analysis, interpretation of data, drafting of the work. GA: acquisition of data, critical revising of the draft. AC, JFM, NH: conception of the work, critical revising of the draft. PBM: conception of the work, acquisition, analysis, interpretation of data, drafting and critical revising of the draft.

Funding MP received a research grant from ADIR association (grant no. ADIR2014-01).

Competing interests MP reports grants from ADIR Association, from B\&D Electromedical, during the conduct of the study; personal fees from Fisher \& Paykel and ResMed, non-financial support from Antadir and Asten, outside the submitted work. JFM reports grants from ADIR Association, during the conduct of the study; personal fees from Philips-Respironics, personal fees from ResMed, personal fees from Air Liquide Santé, personal fees from Breas, personal fees from Boehringer, outside the submitted work. NH reports grants from Guy's and St Thomas' Charity, during the conduct of the study; grants from Philips-Respironics, non-financial support from Philips-Respironics RT Meeting (MYOTRACE), personal fees from Fisher \& Paykel, grants from ResMed, grants from B\&D Electromedical, grants from Fisher \& Paykel, outside the submitted work. In addition, NH has a patent MYOTRACE pending and MP is on the Pulmonary Research Advisory Board for Philips. NH's research group has received unrestricted grants (managed by Guy's and St Thomas' Foundation Trust) from Philips-Respironics, Philips, ResMed, Fisher \& Paykel, and B\&D Electromedical. Philips-Respironics are contributing to the development of the MYOTRACE technology. PBM reports grants and personal fees from Philips-Respironics, grants from ResMed, grants from B\&D Electromedical, outside the submitted work. Other authors have no competing interests to declare.

Patient consent Obtained.

Ethics approval Guy's Research Ethics Committee.

Provenance and peer review Not commissioned; externally peer reviewed.

Author note MP is the guarantor of the content of the manuscript, including the data and analysis. (c) Article author(s) (or their employer(s) unless otherwise stated in the text of the article) 2019. All rights reserved. No commercial use is permitted unless otherwise expressly granted.

\section{REFERENCES}

1 Lacedonia D, Carpagnano GE, Aliani M, et al. Daytime PaO2 in OSAS, COPD and the combination of the two (overlap syndrome). Respir Med 2013;107:310-6.

2 Marin JM, Soriano JB, Carrizo SJ, et al. Outcomes in patients with chronic obstructive pulmonary disease and obstructive sleep apnea: the overlap syndrome. Am J Respir Crit Care Med 2010;182:325-31.

3 Kushida CA, Littner MR, Morgenthaler T, et al. Practice parameters for the indications for polysomnography and related procedures: an update for 2005. Sleep 2005:28:499-523.

4 Mandal S, Arbane G, Murphy P, et al. Medium-term cost-effectiveness of an automated non-invasive ventilation outpatient set-up versus a standard fixed level non-invasive ventilation inpatient set-up in obese patients with chronic respiratory failure: a protocol description. BMJ Open 2015:5:e007082

5 Murphy PB, Rehal S, Arbane G, et al. Effect of home noninvasive ventilation with oxygen therapy vs oxygen therapy alone on hospital readmission or death after an acute COPD exacerbation: a randomized clinical trial. JAMA 2017;317:2177-86.

6 Murphy PB, Arbane G, Ramsay M, et al. Safety and efficacy of auto-titrating noninvasive ventilation in COPD and obstructive sleep apnoea overlap syndrome. Eur Respir J 2015;46:548-51.

7 Global Initiative for Chronic Obstructive Lung Disease (GOLD). From the globa strategy for the diagnosis, management and prevention of COPD. 2017. http:// goldcopd.org

8 Murphy PB, Davidson C, Hind MD, et al. Volume targeted versus pressure support non-invasive ventilation in patients with super obesity and chronic respiratory failure: a randomised controlled trial. Thorax 2012;67:727-34.

9 Struik FM, Lacasse Y, Goldstein RS, et al. Nocturnal noninvasive positive pressure ventilation in stable COPD: a systematic review and individual patient data metaanalysis. Respir Med 2014;108:329-37.

10 Contal $\mathrm{O}$, Vignaux L, Combescure C, et al. Monitoring of noninvasive ventilation by built-in software of home bilevel ventilators: a bench study. Chest 2012;141:469-76.

11 Georges M, Adler D, Contal O, et al. Reliability of apnea-hypopnea index measured by a home bi-level pressure support ventilator versus a polysomnographic assessment. Respir Care 2015:60:1051-6. 